Mexico. Microb Drug Resist 2003;9:33-38.

10. Bobadilla-del-Valle M, Ponce-de-Leon A, Arenas-Huertero C, Vargas-Alarcon G, KatoMaeda M, Small PM, et al. rpoB Gene mutations in rifampin-resistant Mycobacterium tuberculosis identified by polymerase chain reaction singlestranded conformational polymorphism. Emerg Infect Dis 2001;7:1010-1013.

II. Molina-Torres CA, Moreno-Torres E, Ocampo-Candiani J, Rendon A, Blackwood K, Kremer K, et al. Mycobacterium tuberculosis spoligotypes in Monterrey, Mexico. J Clin Microbiol 2010;48:448-455.

12. Garza-Gonzalez E, Gonzalez GM, Renteria A, Cruz-Pulido W, Rivera G, Bocanegra-Garcia V.

A pyrosequencing method for molecular monitoring of regions in the inhA, ahpC and rpoB genes of Mycobacterium tuberculosis. Clin Microbiol Infect 2010;16:607-612.

13. Zenteno-Cuevas R, Zenteno JC, Cuellar A, Cuevas B, Sampieri CL, Riviera JE, et al. Mutations in $r p o B$ and katG genes in Mycobacterium isolates from the Southeast of Mexico. Mem Inst Oswaldo Cruz 2009; 104:468-472.

14. Ramaswamy SV, Dou SJ, Rendon A, Yang Z, Cave MD, Graviss EA. Genotypic analysis of multidrug-resistant Mycobacterium tuberculosis isolates from Monterrey, Mexico. J Med Microbiol 2004;53:107-I I3.

15. Jin DJ, Gross CA. Mapping and sequencing of mutations in the Escherichia coli rpoB gene that lead to rifampicin resistance. J Mol Biol 1988;202:45-58.

16. Telenti A, Imboden P, Marchesi F, Lowrie D, Cole S, Colston MJ, et al. Detection of rifampicin-resistance mutations in Mycobacterium tuberculosis. Lancet 1993;341:647-650. 17. Heep M, Brandstatter B, Rieger U, Lehn $\mathrm{N}$, Richter E, Rusch-Gerdes S, et al. Frequency of $r p \circ B$ mutations inside and outside the cluster I region in rifampin-resistant clinical Mycobacterium tuberculosis isolates. J Clin Microbiol 200 I;39:107-II0.

\section{Respuesta al comentario al artículo "Mutaciones asociadas con resistencia a rifampicina $\odot$ isoniazida en aislamientos clínicos de M. Tuberculosis de Sonora, México"}

Señor editor: Por este medio, los abajo firmantes y autores del trabajo titulado "Mutaciones asociadas con resistencia a rifampicina o isoniazida en aislamientos clínicos de M. tuberculosis de Sonora, Méxi$\mathrm{co}^{\prime \prime}$, le informamos que después de revisar y discutir la carta enviada por Flores-Tre- viño Samantha y Garza-González Elvira, así como la literatura correspondiente, expresamos nuestra aceptación a sus observaciones, por lo que en lo sucesivo utilizaremos ambas clasificaciones moleculares para la discusión de nuestros resultados. No obstante lo anterior, destacamos que la finalidad de nuestro trabajo, más allá de la intención de profundizar en la identificación de mutaciones específicas, fue iniciar la caracterización genotípica de cepas clínicas de M. tuberculosis resistentes a isoniazida o rifampicina aisladas en Sonora, México, ${ }^{1}$ principalmente para fortalecer el monitoreo de la tuberculosis multidrogorresistente ${ }^{2}$ en nuestro país. El otro aspecto relevante de nuestro trabajo es que al analizar seis aislamientos clínicos en los que no se obtuvo un perfil fenotípico de resistencia a fármacos, dos de ellos presentaron mutaciones asociadas con resistencia a isoniazida o rifampicina. De igual manera, destacamos que en cuatro de las cepas no se detectaron mutaciones asociadas con resistencia a esos fármacos, dentro de las regiones analizadas y más frecuentemente evaluadas en este tipo de estudios, ${ }^{3-5}$ incluso para el diseño de nuevos protocolos de evaluación de la farmacorresistencia en $M$. tuberculosis..$^{6-7}$ Finalmente, consideramos que la discusión y conclusiones de nuestro artículo son pertinentes ya que hemos identificado nuevas mutaciones en la región intergénica ahpC-oxyR, particularmente en una región (-12 a -17), región en donde se han detectado mutaciones asociadas con perfiles de resistencia a isoniazida. ${ }^{5}$ En virtud de lo antes expuesto, consideramos que es recomendable realizar la caracterización genotípica sistemática de todas las cepas clínicas de M. tuberculosis que presenten resistencia a cualquier fármaco antituberculoso, en Sonora, México y en otras regiones geográficas del país.

Enrique Bolado-Martínez, D en C(I) ebolado@guayacan.uson.mx Anxis Pérez-Mendoza, $M$ en $C_{\text {, }}^{(2)}$ Francisco Monserrat Alegria-Morquecho, QBC, (3) María del Carmen Candia-Plata, D en $C_{\text {, }}^{(4)}$ María del Rosario Aguayo-Verdugo, QB, (3) Gerardo Álvarez-Hernández, D en C. ${ }^{(4)}$

(I) Departamento de Ciencias Químico Biológicas,
Universidad de Sonora. Hermosillo, Sonora, México

(2) Doctorado Institucional en Ciencias de la Salud, Universidad Autónoma de Yucatán. Yucatán, México

(3) Laboratorio Estatal de Salud Pública. Hermosillo, Sonora. México.

(4) Departamento de Medicina y Ciencias de la Salud, Universidad de Sonora. Hermosillo, Sonora, México

\section{Referencias}

I. Bolado-Martinez E, Perez-Mendoza A, Alegria-Morquecho FM, Candia-Plata M del C, Aguayo-Verdugo M del R, Alvarez-Hernandez G. DNA mutations associated to rifampicin or isoniazid resistance in $\mathrm{M}$. tuberculosis clinical isolates from Sonora, Mexico. Salud Publica Mex 20I2;54:167-I70.

2. WHO. Implementing the Stop TB strategy: a handbook for national tuberculosis control programmes. Geneva: World Health Organization, 2008 (WHO/HTM/TB/2008.40I).

3. Luo T, Zhao M, Li X, Xu P, Gui X, Pickerill S, et $a$ l. Selection of mutations to detect multidrugresistant Mycobacterium tuberculosis strains in Shanghai, China. Antimicrob Agents Chemother 2010;54:1075-1081.

4. Kozhamkulov U, Akhmetova A, Rakhimova S, Belova E, Alenova A, Bismilda V, et al. Molecular characterization of rifampicin- and isoniazid-resistant Mycobacterium tuberculosis strains isolated in Kazakhstan. Jpn J Infect Dis 20I I;64:253-255.

5. Therese KL, Gayathri R, Balasubramanian S, Natrajan S, Madhavan HN. Phenotypic and genotypic characteristics of drug resistance in Mycobacterium tuberculosis isolates from pediatric population of Chennai, India. Indian J Med Microbiol 2012;30:4 II-4I7

6. Bang $\mathrm{H}$, Park S, Hwang J, Jin $\mathrm{H}$, Cho E, Kim DY, et al. Improved rapid molecular diagnosis of multidrug-resistant tuberculosis using a new reverse hybridization assay, REBA MTB-MDR. J Med Microbiol 201 I;60: I447- I454.

7. Daum LT, Rodriguez JD, Worthy SA, Ismail NA, Omar SV, Dreyer AW, et al. Next-generation ion torrent sequencing of drug resistance mutations in Mycobacterium tuberculosis Strains. J Clin Microbiol 2012;50:383I-3837.

\section{Letalidad por fiebre manchada por Rickettsia rickettsii en pacientes de un hospital pediátrico del estado de Sonora, 2004-20 I 2}

La fiebre manchada por Rickettsia rickettsii (FMRR) es la más letal de las infecciones del grupo de fiebres manchadas. ${ }^{1-2} \mathrm{Si}$ bien la enfermedad puede ocurrir en cualquier 
persona, dos tercios de los casos se presentan en sujetos menores de 15 años $^{3}$ y la mayor incidencia tiene lugar en los niños entre 5 y 9 años, ${ }^{4}$ quienes tienen el mayor riesgo de complicaciones, y hasta $3 \%$ de ellos fallecen actualmente en Estados Unidos. ${ }^{5}$ En Sonora, durante el último lustro se registran aproximadamente 100 casos del padecimiento cada año, y la letalidad en la población general oscila entre $8 \mathrm{y}$ $17.8 \% .^{6}$ En el Hospital Infantil del Estado de Sonora (HIES) desde el año 2004 se han registrado 121 casos de FMRR y 35 de esos niños han fallecido, con una letalidad anual que fluctúa entre 0 y $63 \%$, y una tendencia ascendente desde la remergencia de la enfermedad a inicios de la década.

Diversos factores concurren para explicar la letalidad de FMRR; algunos pueden relacionarse con el inóculo y virulencia de la cepa de $R$. rickettsii, con patrones de alimentación de la garrapata transmisora, con variaciones geográficas y con la edad, sexo y otros factores biológicos del huésped, ${ }^{1-2,7}$ pero sin duda dos factores de la atención médica son determinantes de resultados fatales: la pobre oportunidad diagnóstica y el retraso (mayor a cinco días tras el inicio de síntomas) en el comienzo del tratamiento con doxiciclina..$^{8-9}$ Existe, en general, un temor infundado para prescribir la doxiciclina, el antibiótico de elección, a pacientes pediátricos, hecho indeseable que ocurre con frecuencia incluso en regiones endémicas de Estados Unidos. ${ }^{10-11}$

Además, en nuestra experiencia, el desconocimiento del personal médico contribuye de modo decisivo en la ocurrencia de casos severos de la enfermedad, pues 90\% de los niños atendidos en este hospital recibió al menos dos consultas médicas previas, y en 3 de cada 4 pacientes se les diagnosticó y trató como una faringitis aguda infecciosa. Si consideramos también que los pacientes son referidos en condiciones de extrema gravedad y con inestabilidad hemodinámica y metabólica, se puede entender que la muerte del menor ocurra tras únicamente 1.6 días de estancia hospitalaria y con sólo dos dosis de doxiciclina, sin oportunidad suficiente para la acción farmacológica del antibiótico ni respuesta a las medidas de soporte res- piratorio y aminérgico. El desconocimiento del personal médico es crucial y debe ser motivo de estrategias educativas efectivas para disminuir el negativo impacto que provoca. Esto no es exclusivo de Sonora, pues en Estados Unidos se ha documentado que sólo 1 de cada 4 médicos identifican correctamente al padecimiento e inician el tratamiento con doxiciclina. ${ }^{12}$

La letalidad por FMMR en pacientes pediátricos atendidos en el HIES es mayor a cualquier otro padecimiento infeccioso de interés epidemiológico como tosferina, influenza A H1N1, tuberculosis, neumonías y diarrea infecciosa, y en nuestra opinión debe considerarse como una emergencia sanitaria y un problema prioritario de salud pública en el estado, algo que ya hemos sugerido incluso previamente. ${ }^{13}$ De hecho, el comportamiento actual en Sonora es similar al que se apreciaba en regiones endémicas de Estados Unidos en la década de los 40 y 50, ${ }^{14}$ aunque es posible que también ocurran casos en otros estados del país y que la carga del padecimiento esté subestimada, tal como sucede en otras regiones endémicas. ${ }^{15}$

Nuestra recomendación es que se discutan estrategias de contención y prevención de la letalidad por FMRR, tanto al interior de los hospitales como en unidades de primer nivel de atención, específicamente para mejorar: 1) la sospecha diagnóstica y el inicio temprano (primeras 72 horas) de doxiciclina; 2) la disponibilidad de doxiciclina en presentación oral en unidades de primer nivel de atención, así como oral y parenteral en hospitales; 3) la referencia oportuna y adecuada de pacientes hospitalizados; 4) el manejo hospitalario de la sepsis, choque séptico y otras complicaciones metabólicas y hemodinámicas asociadas con casos graves de FMRR; 5) la identificación de factores de riesgo y mal pronóstico en pacientes pediátricos con FMRR.

\section{Gerardo Álvarez Hernández, PhD, (I) galvarezh63@gmail.com José Jesús Contreras Soto, $M$ en $C^{(2)}$}

(I) Jefe de la Unidad de Vigilancia Epidemiológica Hospitalaria, Hospital Infantil del Estado de Sonora. Sonora, México

(2) Dirección Médica, Hospital Infantil del Estado de Sonora. Sonora, México

\section{Referencias}

I. Dantas-Torres F. Rocky Mountain Spotted Fever. Lancet Infect Dis 2007; 7: 724-732. 2. Chen LF, Sexton DJ. What's new in Rocky Mountain Spotted Fever? Infect Dis Clin N Am 2008; 22: 415-432.

3. Razzaq S, Schutze GE. Rocky Mountain Spotted Fever: a physician's challenge. Pediatr Rev 2005; 26: I25-130.

4. Treadwell TA, Holman RC, Clarke MJ, Krebs JW, Paddock CD, Childs JE. Rocky Mountain Spotted Fever in the United States, 1993-1996. Am J Trop Med Hyg 2000; 63 (I): 2I-26. 5. Openshaw JJ, Swerdlow DL, Krebs JW, Holman RC, Mandel E, Harvey A, et al. Rocky Mountain Spotted Fever in the United States, 2000-2007: Interpreting contemporary increases in Incidence. Am J Trop Med Hyg 2010; 83(I):I74-I82.

6. Secretaría de Salud Pública del Estado de Sonora. Caracterización de la Fiebre Manchada debida a Rickettsia rickettsii, a la semana epidemiológica 36, 2012. Dirección General de Servicios de Salud a la Comunidad/Unidad de Inteligencia Epidemiológica y Emergencias en Salud. México: Secretaría de Salud Pública del Estado de Sonora, 2012.

7. Walker DH. Editorial: Rickettsia rickettsii as virulent as ever. Am J Trop Med Hyg 2002; 66 (5): 448-449.

8. Kirkland KB, Wilkinson WE, Sexton TJ. Therapeutic delay and mortality in cases of Rocky Mountain Spotted Fever. Clin Inf Dis 1995; 20 (5): III8-I|2I.

9. Buckingham SC, Marshall GS, Schutze GE, Woods CR, Jackson MA, Patterson LER, et al. Clinical and laboratory features, hospital course, and outcome of Rocky Mountain Spotted Fever in children. J Pediatr 2007; 150: 180-184.

10. Purvis J, Edwards MS. Doxycycline use for rickettsial disease in pediatric patients. Pediatr Dis Infect J 2000; 19 (9): 87I-874.

II. Masters EJ, Olson GS, Weiner SJ, Paddock CD. Rocky Mountain Spotted Fever. A clinician's dilemma. Arch Intern Med 2003; 163: 769-774.

12. O'Reilly M, Paddock C, Elchos B, Goddard J, Childs J, Currie M. Physician knowledge of the diagnosis and management of Rocky Mountain Spotted Fever. Mississippi 2002. Ann NY Acad Sci 2003; 990: 295-30I.

13. Álvarez-Hernández G. La Fiebre Manchada de las Montañas Rocosas, una epidemia olvidada. Salud Publica Mex 20I0; 52 (2): I-3.

14. Childs JE, Paddock CD. Rocky Mountain Spotted Fever. In: Parola P, Raoult D. (Eds.) Rickettsial Diseases. Informa Health Care. France, 2007: 97- I |6ISBN: 0849376 I |4.

15. Paddock CD, Greer PW, Ferebee TL, Singleton J, McKechnie DB, Treadwell TA. Hidden mortality attributable to Rocky Mountain Spotted Fever: immunohistochemical detection of fatal, serologically unconfirmed cases. J Infect Dis 1999; 179: 1469-| 478. 